\title{
Characteristic Analysis of Fractional-Order 4D Hyperchaotic Memristive Circuit
}

\author{
Jun Mou, ${ }^{1,2}$ Kehui Sun, ${ }^{1}$ Huihai Wang, ${ }^{1}$ and Jingya Ruan' \\ ${ }^{1}$ School of Physics and Electronics, Central South University, Changsha 410083, China \\ ${ }^{2}$ School of Information Science and Engineering, Dalian Polytechnic University, Dalian 116034, China \\ Correspondence should be addressed to Kehui Sun; kehui@csu.edu.cn
}

Received 6 March 2017; Accepted 28 May 2017; Published 10 July 2017

Academic Editor: Alessandro Lo Schiavo

Copyright (C) 2017 Jun Mou et al. This is an open access article distributed under the Creative Commons Attribution License, which permits unrestricted use, distribution, and reproduction in any medium, provided the original work is properly cited.

Dynamical behaviors of the 4D hyperchaotic memristive circuit are analyzed with the system parameter. Based on the definitions of fractional-order differential and Adomian decomposition algorithm, the numerical solution of fractional-order 4D hyperchaotic memristive circuit is investigated. The distribution of stable and unstable regions of the fractional-order 4D hyperchaotic memristive circuit is determined, and dynamical characteristics are studied by phase portraits, Lyapunov exponents spectrum, and bifurcation diagram. Complexities are calculated by employing the spectral entropy (SE) algorithm and $C_{0}$ algorithm. Complexity results are consistent with that of the bifurcation diagrams, and this means that complexity can also reflect the dynamic characteristics of a chaotic system. Results of this paper provide a theoretical and experimental basis for the application of fractionalorder $4 \mathrm{D}$ hyperchaotic memristive circuit in the field of encryption and secure communication.

\section{Introduction}

According to the principle of completeness with variable combination, Professor Chua predicted the existence of memristor in 1971 [1]. In 1976, he expounded the character of memristor, composition principle, and applications [2]. For a long time, the existence of element which satisfied the character of memristor was not discovered, so the study of memristor did not rise to the attention of scientific community and engineering circles. In 2008, the HP laboratory reported the realization of memristor firstly $[3,4]$, and, since then, the memristor has attracted much attention all over the world. Memristors are often divided into chargecontrolled memristor and flux-controlled memristor. Both of them are typical nonlinear elements. It is expected to be an effective memory storage device in computers. It means lower power consumption and less thermal design to deal with, and it is easy to generate a chaotic vibration signal by employing this element. On one hand, we need to prevent the harm of chaos phenomenon in the application. On the other hand, the chaotic vibration signal by employing the memristor can be applied to many fields such as secure communication and aerospace industry. So researchers began to focus on the design and realization of memristive chaotic circuit [5-20]. In these literatures, only one memristor was applied in an independent circuit, and the dynamic characteristics of memristive chaotic system are related to the initial state of memristor, including unique nonlinear physics phenomenon. Zhang and Deng studied double-compound synchronization of six memristor-based Lorenz systems [21]. However, this article focuses on the synchronization method of a net with four Lorenz systems, rather than dynamic behaviors of a memristive circuit. When Bao et al. and Mou et al. applied more than one memristor in a single circuit [22, 23], they found that memristors would affect each other, and the dynamical behaviors of the circuit with more than one memristor become more complex. Compared with ordinary memristive chaotic systems, the memristive hyperchaotic systems have excellent security in communication because the memristive hyperchaotic sequence has better complexity and randomicity [24-26].

Fractional calculus is more than 300-year-old topic. It can describe physical phenomena more accurately than that of the integer-order calculus, so fractional calculus has attracted more attention in various areas of applications such as physics, chemistry, bioengineering, signal processing, and 
control system [27-29]. A very important area of applications is the chaos theory [30-33]. At present, there are three typical methods to solve the fractional-order nonlinear system, such as frequency domain approximation [30], predictorcorrector method (PCM) [34], and Adomian decomposition method (ADM) [35]. The calculation precision of frequency domain approximations is limited. Whether this method accurately reflects the chaos characteristics of a fractionalorder nonlinear system was questioned [36, 37]. For PCM, one can obtain more accurate results, but the calculation speed is too slow, and it consumes too much computer resources. Thus, it is unsuitable for engineering practice. Compared with the two solution approaches, ADM is capable of dealing with linear and nonlinear problems in time domain $[38,39]$. He et al. concluded the characteristics of this method, such as high accuracy, fast convergence, and less computer resources consumption [40]. Recently, Li et al. proposed a new 4D hyperchaotic memristive circuit [41], which possesses abundant complex dynamics. The main feature of this system is having uncountable infinite number of stable equilibria, which is significantly different from other reported chaotic systems before. However, the article mainly analyzed the equilibrium states, and the analysis of dynamics characteristics of the $4 \mathrm{D}$ memristive hyperchaotic system is incomplete. Its fractional-order form has not been studied by now. Thus, it makes a great sense to study the dynamics characteristics of the system and the solution and dynamics of its corresponding fractional-order case. To our knowledge, no one has studied the fractional-order memristive hyperchaotic system. So we will employ ADM algorithm to solve the fractional-order 4D hyperchaotic system.

In this paper, we focus on dynamical characteristic of the fractional-order 4D hyperchaotic memristive circuit. It is organized as follows. The dynamical characteristics of integer-order 4D hyperchaotic memristive circuit are investigated in Section 2. In Section 3, ADM is introduced briefly, and the iterative algorithm of the fractional-order $4 \mathrm{D}$ hyperchaotic memristive circuit is deduced. In Section 4, the distribution of stable and unstable regions of the fractionalorder $4 \mathrm{D}$ hyperchaotic memristive circuit is determined, and the dynamical characteristics of this system are analyzed. Finally, we summarize the results and indicate future directions.

\section{4D Hyperchaotic Memristive Circuit}

2.1. Model of the 4D Hyperchaotic Memristive Circuit. Figure 1 shows the 4D hyperchaotic memristive circuit model, which consists of standard integrators, standard multipliers, linear resistors, linear capacitors, and a nonlinear active memristor. We can use $x, y$, and $z$ to indicate states of voltages. For the memristive circuit, there is an input from $x$ to $y$ by a flux-controlled memristor, and it is illustrated by $W$ in Figure 1. According to Chua's definition, a memristor is a passive two-terminal circuit element described by a nonlinear $i-v$ characteristic as follows: $v=M(z) i$ or $i=W(\varphi) v$, where $v, i, z$, and $\varphi$ are the voltage, current, charge, and flux associated with the device. $M(z)$ is the memristance defined as follows: $M(z)=d \varphi(z) / d z \cdot W(\varphi)$ is the memductance

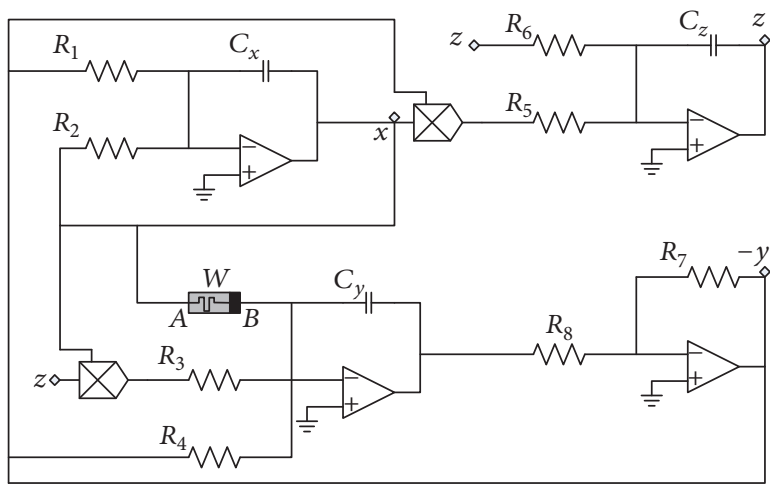

FIGURE 1: The 4D hyperchaotic memristive circuit.

defined as follows: $W(\varphi)=d z(\varphi) / d \varphi$. Here, we focus on the flux-controlled memristive system described by the following circuit equation: $W(\varphi)=a+3 b \varphi^{2}$, where $a$ and $b$ are two positive constant parameters. According to the volt-ampere characteristics of each element and Kirchhoff's current and voltage law, the differential equation (1) is obtained:

$$
\begin{aligned}
C_{x} \dot{x} & =\frac{y}{R_{1}}-\frac{x}{R_{2}} \\
C_{y} \dot{y} & =-\frac{x z}{R_{3}}+\frac{y}{R_{4}}-W(\varphi) x \\
C_{z} \dot{z} & =\frac{x y}{R_{5}}-\frac{z}{R_{6}} \\
\dot{\varphi} & =x,
\end{aligned}
$$

where $R$ is a reference resistor and $C$ is a reference capacitor; then $t=R C$ is the physical time, where $t$ is the dimensionless time. The parameters can be taken as follows: $C_{x}=C_{y}=C_{z}=$ $C, R_{3}=R_{5}=R, \alpha=R / R_{1}=R / R_{2}, \beta=R / R_{4}$, and $\gamma=R / R_{6}$. By employing the normalized operation, (1) becomes

$$
\begin{aligned}
& \dot{x}=\alpha(y-x) \\
& \dot{y}=-x z+\beta y-\rho W(w) x \\
& \dot{z}=x y-\gamma z \\
& \dot{w}=x,
\end{aligned}
$$

where $\rho$ is a positive parameter indicating the strength of the memristor. We should note here that $W(w)$ comes from the memristor and has the same function as the physical memductance mentioned above, but it is dimensionless, which would be convenient for the following discussion.

Setting the parameters $a=4, b=0.01, \alpha=36, \beta=20$, $\gamma=3, \rho=3$, the initial value of $(2)$ is $(1,0,1,0)$, and the time step is $t=0.001 \mathrm{~s}$; we get the hyperchaotic attractor as shown in Figure 2. In this case, the Lyapunov exponents of the system are $L_{1}=0.2566, L_{2}=0.0674$, and $L_{3}=0, L_{4}=-19.2935$, and the Lyapunov dimension is $D_{L}=3.017$. Obviously the first two positive Lyapunov exponents imply that the $4 \mathrm{D}$ memristive circuit is hyperchaotic. 


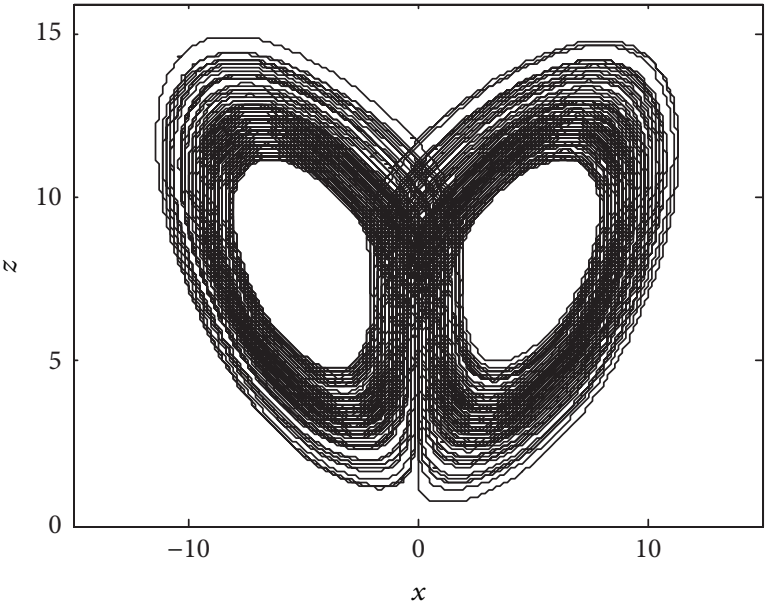

(a)

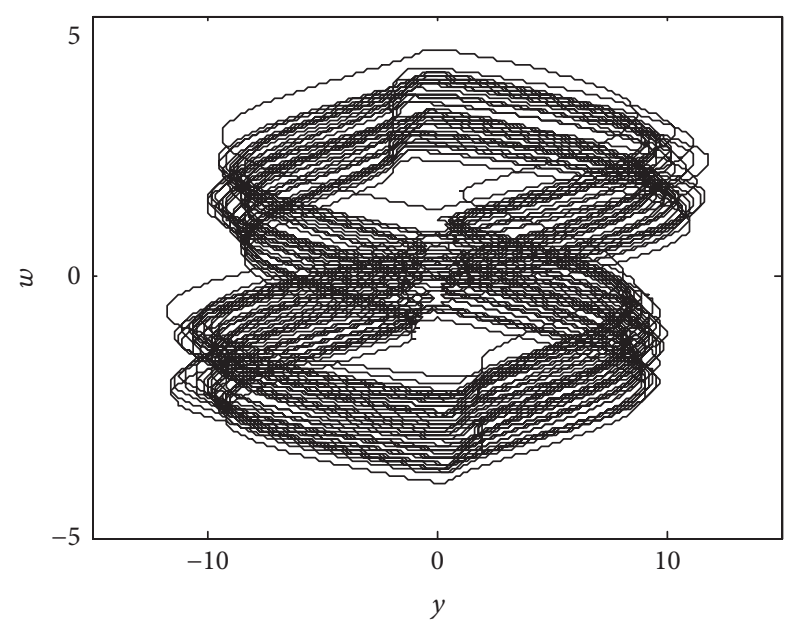

(b)

FIGURE 2: Hyperchaotic attractor of the 4D hyperchaotic memristive circuit: (a) $x-z$ and (b) $y-w$.

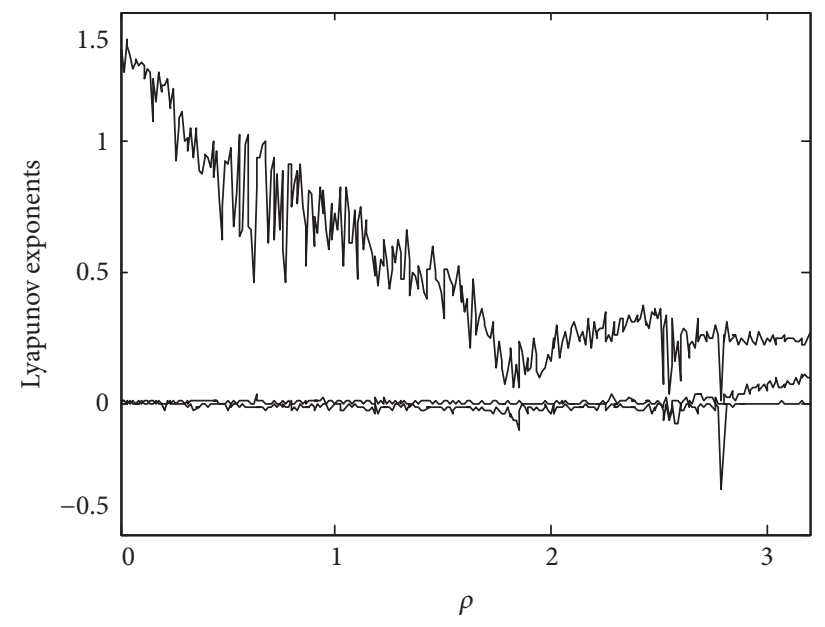

(a) Lyapunov exponents spectrum

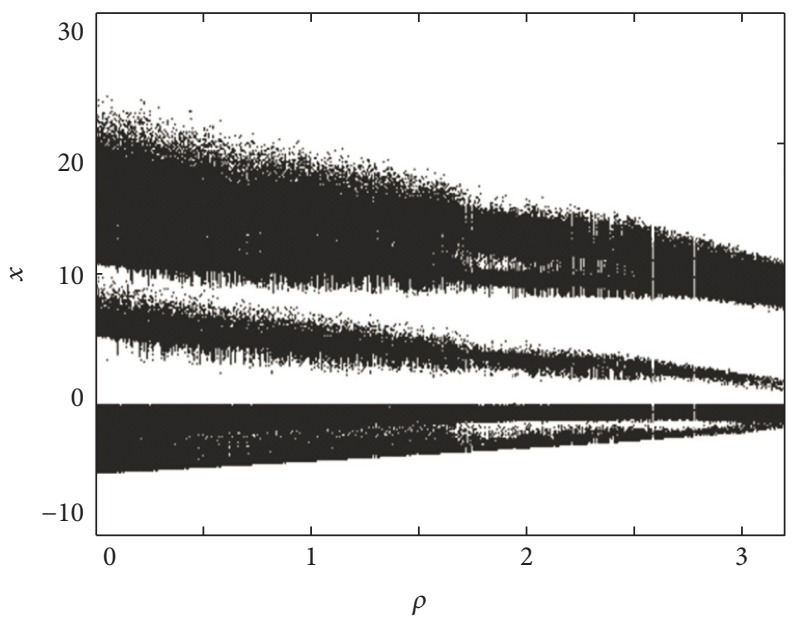

(b) Bifurcation diagram

FIGURE 3: Lyapunov exponents spectrum and bifurcation diagram with variation of the parameter $\rho$.

2.2. Dynamic Analysis with Different System Parameter. Fix the initial value of $(2)$ to $(1,0,1,0)$, and change the parameter $\rho$ from 0 to 3.2. Other parameters are the same as mentioned above. We obtain the Lyapunov exponents and its corresponding bifurcation diagram as shown in Figure 3, where the last Lyapunov exponent is not displayed because it is always a big negative number. It shows that the Lyapunov exponents spectrum and bifurcation diagram are consistent. Figure 3(b) shows the routes to chaos of the system (2), and the system transforms into two-scroll hyperchaotic attractor from one-scroll chaotic attractor. When the circuit parameter $\rho$ increases further, the system transforms into three-scroll hyperchaotic attractor from two-scroll hyperchaotic attractor. Obviously, there are two periodic windows in the chaotic region at about $\rho=2.55$ and $\rho=2.79$. It indicates that system (2) has abundant dynamical behaviors.

To display its dynamics further, $y-w$ phase portraits of different states with different parameter $\rho$ are presented in Figure 4 .

\section{Numerical Solution of Fractional-Order 4D Hyperchaotic Memristive Circuit}

3.1. Adomian Decomposition Method. For a given fractionalorder differential equation ${ }^{*} D_{t 0}^{q}(t)=f(x(t))$, here $x(t)=$ $\left[x_{1}(t), x_{2}(t), \ldots, x_{n}(t)\right]^{T}$ are variables and ${ }^{*} D_{t 0}^{q}(t)$ is the Caputo derivative operator of order $q[42,43]$. To obtain the following initial value problem, $f(x(t))$ is separated into three terms $[44,45]$ :

$$
\begin{aligned}
{ }^{*} D_{t 0}^{q} x(t) & =L x(t)+N x(t)+g(t) \\
x^{(k)}\left(t_{0}^{+}\right) & =b_{k}, \quad k=0,1, \ldots, m-1 .
\end{aligned}
$$

Here, $L$ and $N$ represent linear and nonlinear items, respectively, and $g(t)=\left[g_{1}(t), g_{2}(t), \ldots, g_{n}(t)\right]^{T}$ are constants for autonomous systems, and $b_{k}$ is a specified constant. 


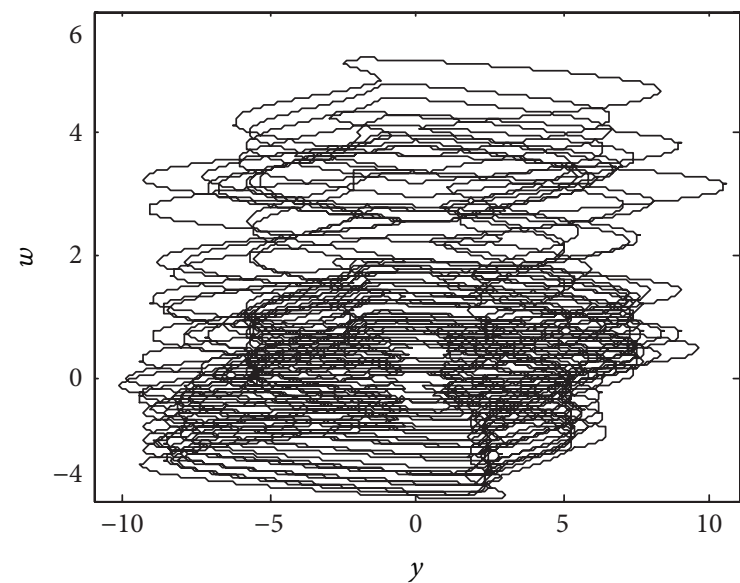

(a) Chaotic attractor $(\rho=0.5)$

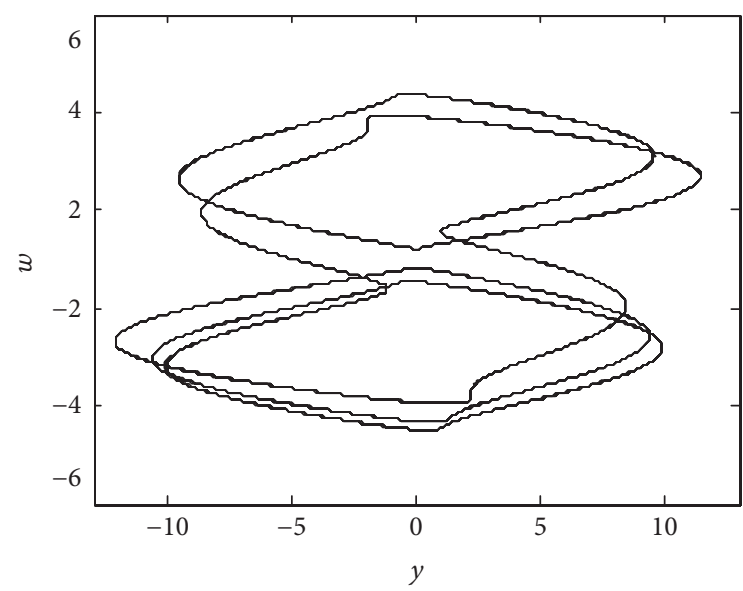

(c) Type 2 limit cycle $(\rho=2.79)$

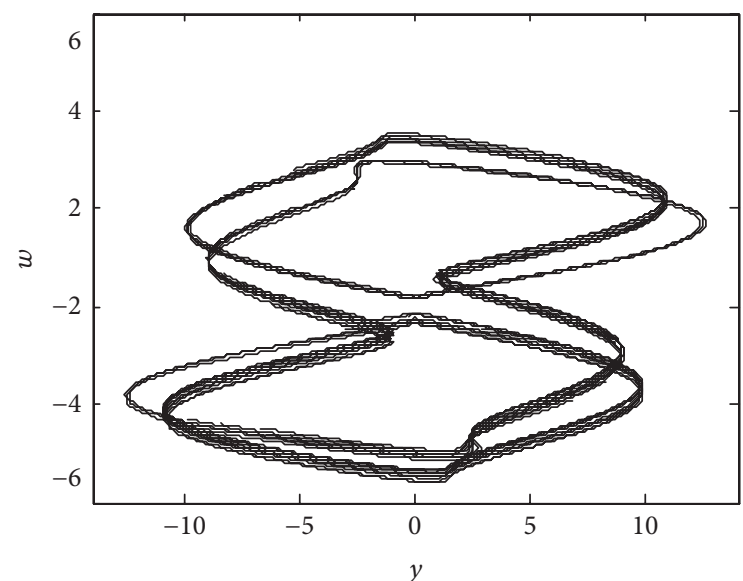

(b) Type 1 limit cycle $(\rho=2.55)$

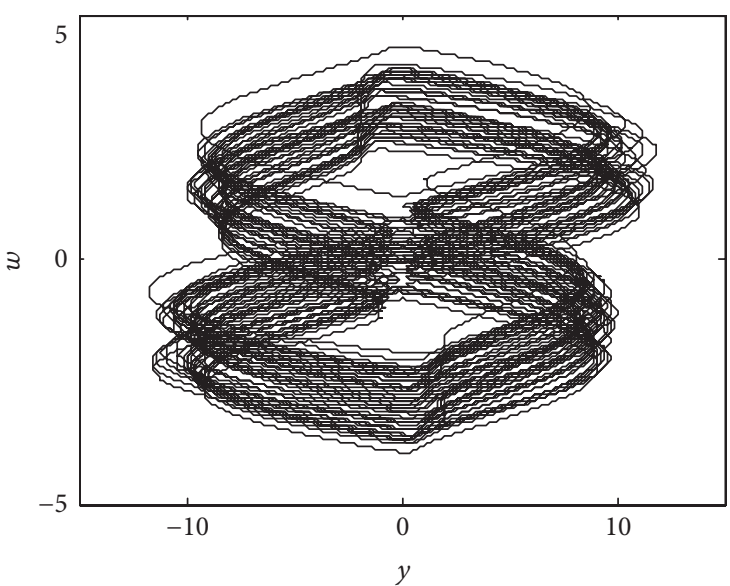

(d) Two-scroll hyperchaotic attractor $(\rho=3)$

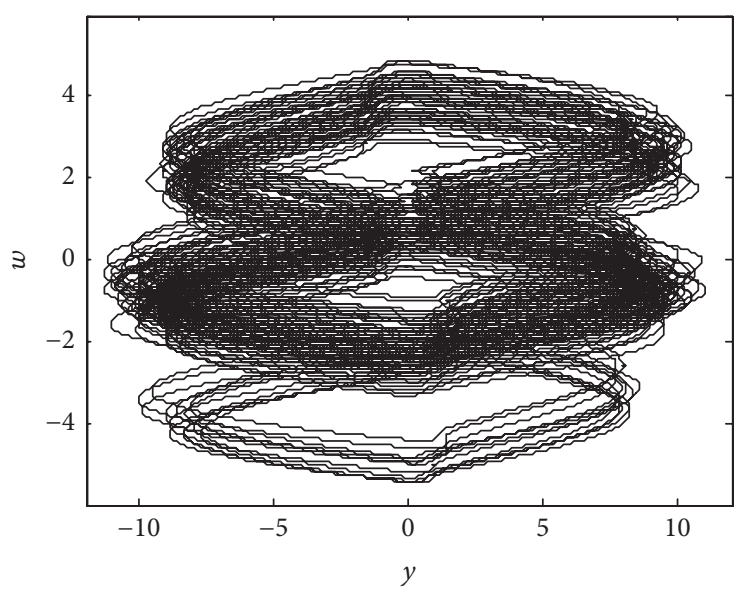

(e) Three-scroll hyperchaotic attractor $(\rho=3.15)$

FIGURE 4: Phase portraits with different parameter $\rho$.

By applying the operator $J_{t 0}^{q}$ to both sides of (3), the following equation is obtained [46]:

$$
x=J_{t 0}^{q} L x+J_{t 0}^{q} N x+J_{t 0}^{q} g+\sum_{k=0}^{m-1} b_{k} \frac{\left(t-t_{0}\right)^{k}}{k !} .
$$

$J_{t 0}^{q}$ is Riemann-Liouville fractional integral operator with order $q$. For $t=\left[t_{0}, t_{1}\right], q \geqslant 0, r \geqslant 0, \gamma>-1$, and real constant $C$, the fundamental properties of the integral operator $J_{t 0}^{q}$ are described as follows [47]: 


$$
\begin{aligned}
J_{t 0}^{q}\left(t-t_{0}\right)^{\gamma} & =\frac{\Gamma(\gamma+1)}{\Gamma(\gamma+1+q)}\left(t-t_{0}\right)^{\gamma+q}, \\
J_{t 0}^{q} C & =\frac{C}{\Gamma(q+1)}\left(t-t_{0}\right)^{q}, \\
J_{t 0}^{q} J_{t 0}^{r} x(t) & =J_{t 0}^{q+r} x(t) .
\end{aligned}
$$

Based on ADM, the nonlinear terms of (4) are decomposed according to

$$
\begin{aligned}
A_{j}^{i} & =\frac{1}{i !}\left[\frac{d^{i}}{d \lambda^{i}} N\left(v_{j}^{i}(\lambda)\right)\right]_{\lambda=0} \\
v_{j}^{i}(\lambda) & =\sum_{k=0}^{i}(\lambda)^{k} x_{j}^{k},
\end{aligned}
$$

where $i=0,1, \ldots, \infty, j=1,2, \ldots, n$; then the nonlinear terms are expressed as

$$
N x=\sum_{i=0}^{\infty} A^{i}\left(x^{0}, x^{1}, \ldots, x^{i}\right)
$$

So the solution of (3) $x=\sum_{i=0}^{\infty} x^{i}$ is derived from

$$
\begin{gathered}
x^{0}=J_{t 0}^{q} g+\sum_{k=0}^{m-1} b_{k} \frac{\left(t-t_{0}\right)^{k}}{k !} \\
x^{1}=J_{t 0}^{q} L x^{0}+J_{t 0}^{q} A^{0}\left(x^{0}\right) \\
x^{2}=J_{t 0}^{q} L x^{1}+J_{t 0}^{q} A^{1}\left(x^{0}, x^{1}\right) \\
\vdots \\
x^{i}=J_{t 0}^{q} L x^{i-1}+J_{t 0}^{q} A^{i-1}\left(x^{0}, x^{1}, \ldots, x^{i-1}\right) \\
\vdots
\end{gathered}
$$

3.2. Solution of the Fractional-Order 4D Hyperchaotic Memristive Circuit. The equation of the fractional-order $4 \mathrm{D}$ hyperchaotic memristive circuit is

$$
\begin{aligned}
& C_{10}=x_{m} \\
& C_{20}=y_{m} \\
& C_{30}=z_{m} \\
& C_{40}=w_{m} \\
& C_{11}=\alpha\left(C_{20}-C_{10}\right) \\
& C_{21}=-C_{10} C_{30}+\beta C_{20}-a \rho C_{10}-3 b \rho C_{10} C_{40}^{2} \\
& C_{31}=C_{10} C_{20}-\gamma C_{30}
\end{aligned}
$$

$$
\begin{aligned}
& { }^{*} D_{t 0}^{q} x=\alpha(y-x) \\
& { }^{*} D_{t 0}^{q} y=-x z+\beta y-\rho W(w) x \\
& { }^{*} D_{t 0}^{q} z=x y-\gamma z \\
& { }^{*} D_{t 0}^{q} w=x,
\end{aligned}
$$

where $x, y, z$, and $w$ are the state variables, and $q(0<q \leqslant 1)$ is the order of fractional-order differential equation, where $W(\varphi)$ is the memductance defined as $W(w)=a+3 b w^{2}$, and $a, b, \alpha, \beta, \gamma$, and $\rho$ are the system parameters. According to (5) and (8), the discrete iterative formula of the system (9) is presented by

$$
\begin{aligned}
x_{m+1} & =x_{m}+\alpha\left(y_{m}-x_{m}\right) \frac{h^{q}}{\Gamma(q+1)} \\
& +\alpha\left(-x_{m} z_{m}+\beta y_{m}-a \rho x_{m}-3 b \rho x_{m} w_{m}^{2}+\cdots\right) \\
\cdot & \frac{h^{2 q}}{\Gamma(2 q+1)}+\cdots \\
y_{m+1} & =y_{m}+\left(-x_{m} z_{m}+\beta y_{m}-a \rho x_{m}-3 b \rho x_{m} w_{m}^{2}\right) \\
\cdot & \frac{h^{q}}{\Gamma(q+1)}+\left(-x_{m} x_{m} y_{m}+\cdots\right) \frac{h^{2 q}}{\Gamma(2 q+1)}+\cdots \\
z_{m+1} & =z_{m}+\left(x_{m} y_{m}-\gamma z_{m}\right) \frac{h^{q}}{\Gamma(q+1)} \\
& +\left(\alpha\left(y_{m}-x_{m}\right) y_{m}-\gamma\left(x_{m} y_{m}-\gamma z_{m}\right)+\cdots\right) \\
& +\frac{h^{2 q}}{\Gamma(2 q+1)}+\cdots \\
w_{m+1} & =w_{m}+x_{m} \frac{h^{q}}{\Gamma(q+1)}+\alpha\left(y_{m}-x_{m}\right) \frac{h^{2 q}}{\Gamma(2 q+1)} \\
&
\end{aligned}
$$

where $h$ is iteration step size. $\Gamma(\cdot)$ is Gamma function. Considering the fast convergence of this method, we truncate the first six terms of (10) in this paper. For the computer simulation, the iteration is expressed as follows: 


$$
\begin{aligned}
& C_{41}=C_{10} \\
& C_{12}=\alpha\left(C_{21}-C_{11}\right) \\
& C_{22}=-C_{10} C_{31}-C_{11} C_{30}+\beta C_{20}-a \rho C_{11}-3 b \rho\left(C_{11} C_{40}^{2}+2 C_{10} C_{40} C_{41}\right) \\
& C_{32}=C_{11} C_{20}+C_{10} C_{21}-\gamma C_{31} \\
& C_{42}=C_{11} \\
& C_{13}=\alpha\left(C_{22}-C_{12}\right) \\
& C_{23}=-C_{10} C_{32}-C_{12} C_{30}-C_{11} C_{31} \frac{\Gamma(2 q+1)}{\Gamma_{q+1}^{2}}+\beta C_{22}-a \rho C_{12} \\
& -3 b \rho\left(C_{12} C_{40}^{2}+2 C_{10} C_{40} C_{42}+\left(2 C_{11} C_{40} C_{41}+C_{10} C_{41}^{2}\right) \frac{\Gamma(2 q+1)}{\Gamma_{q+1}^{2}}\right) \\
& C_{33}=C_{10} C_{22}+C_{12} C_{20}-\gamma C_{32}+C_{11} C_{21} \frac{\Gamma(2 q+1)}{\Gamma_{q+1}^{2}} \\
& C_{43}=C_{12} \\
& C_{14}=\alpha\left(C_{23}-C_{13}\right) \\
& C_{24}=-C_{13} C_{30}-C_{10} C_{33}-\left(C_{12} C_{31}+C_{11} C_{32}\right) \frac{\Gamma(3 q+1)}{\Gamma(q+1) \Gamma(2 q+1)}+\beta C_{23}-a \rho C_{13} \\
& -3 b \rho\left(C_{13} C_{40}^{2}+2 C_{10} C_{40} C_{43}+\left(2 C_{12} C_{40} C_{41}+2 C_{11} C_{40} C_{42}+2 C_{10} C_{41} C_{42}\right) \frac{\Gamma(3 q+1)}{\Gamma(q+1) \Gamma(2 q+1)}\right) \\
& +C_{11} C_{41}^{2} \frac{\Gamma(3 q+1)}{\Gamma_{q+1}^{3}} \\
& C_{34}=C_{13} C_{20}+C_{10} C_{23}+\left(C_{12} C_{21}+C_{11} C_{22}\right) \frac{\Gamma(3 q+1)}{\Gamma(q+1) \Gamma(2 q+1)}-\gamma C_{33} \\
& C_{44}=C_{13} \text {, } \\
& C_{15}=\alpha\left(C_{24}-C_{14}\right) \\
& C_{25}=-C_{14} C_{30}-C_{10} C_{34}-\left(C_{13} C_{31}+C_{11} C_{33}\right) \frac{\Gamma(4 q+1)}{\Gamma(q+1) \Gamma(3 q+1)}-C_{12} C_{32} \frac{\Gamma(4 q+1)}{\Gamma_{2 q+1}^{2}}+\beta C_{24}-a \rho C_{14} \\
& -3 b \rho\left(C_{14} C_{40}^{2}+C_{10} C_{40} C_{44}+\left(2 C_{13} C_{40} C_{41}+2 C_{11} C_{40} C_{43}+2 C_{10} C_{41} C_{43}\right) \frac{\Gamma(4 q+1)}{\Gamma(q+1) \Gamma(3 q+1)}\right) \\
& +\left(2 C_{12} C_{40} C_{42}+C_{10} C_{42}^{2}\right) \frac{\Gamma(4 q+1)}{\Gamma_{2 q+1}^{2}}+\left(C_{12} C_{41}^{2}+2 C_{11} C_{41} C_{42}\right) \frac{\Gamma(4 q+1)}{\Gamma(2 q+1) \Gamma_{q+1}^{2}} \\
& C_{35}=C_{14} C_{20}+C_{10} C_{24}+\left(C_{13} C_{21}+C_{11} C_{23}\right) \frac{\Gamma(4 q+1)}{\Gamma(q+1) \Gamma(3 q+1)}+C_{12} C_{22} \frac{\Gamma(4 q+1)}{\Gamma_{2 q+1}^{2}}-\gamma C_{33} \\
& C_{45}=C_{14} \text {, } \\
& {\left[\begin{array}{c}
x_{m+1} \\
y_{m+1} \\
z_{m+1} \\
w_{m+1}
\end{array}\right]=\left[\begin{array}{llllll}
C_{10} & C_{11} & C_{12} & C_{13} & C_{14} & C_{15} \\
C_{20} & C_{21} & C_{22} & C_{23} & C_{24} & C_{25} \\
C_{30} & C_{31} & C_{32} & C_{33} & C_{34} & C_{35} \\
C_{40} & C_{41} & C_{42} & C_{43} & C_{44} & C_{44}
\end{array}\right]=\left[\begin{array}{llllll}
1 & \frac{h^{q}}{\Gamma(q+1)} & \frac{h^{2 q}}{\Gamma(2 q+1)} & \frac{h^{3 q}}{\Gamma(3 q+1)} & \frac{h^{4 q}}{\Gamma(4 q+1)} & \frac{h^{5 q}}{\Gamma(5 q+1)}
\end{array}\right]^{T}}
\end{aligned}
$$


According to (11), the chaotic sequences of the fractionalorder 4D hyperchaotic memristive circuit are obtained with initial values, $h, q$, and appropriate parameters. Then we can analyze the dynamical characteristics of the system by using the chaotic sequences.

\section{Dynamical Characteristics}

4.1. Stability Analysis. By setting the left-hand side of (9) to zero, we can calculate the equilibrium points of the system. Obviously, $p_{0}=(x, y, z, w)=(0,0,0, n)$ is the one equilibrium point set for the system, $n$ is a real constant. The Jacobian matrix of the system (9) at equilibrium point set $p_{0}$ is described as follows:

$$
J_{\left(P_{0}\right)}=\left[\begin{array}{cccc}
-\alpha & \alpha & 0 & 0 \\
-a \rho-3 \rho b n^{2} & \beta & 0 & 0 \\
0 & 0 & -\gamma & 0 \\
1 & 0 & 0 & 0
\end{array}\right] .
$$

The characteristic polynomial of (12) is $\lambda(\lambda+\gamma)\left(\lambda^{2}+(\alpha-\right.$ $\left.\beta)-\alpha \beta+a \alpha \rho+3 \alpha \rho b n^{2}\right)=0$, which has root at

$$
\begin{aligned}
& \lambda_{1}=0 \\
& \lambda_{2}=-\gamma \\
& \lambda_{2} \\
& =\frac{\left(\beta-\alpha+j \sqrt{4 a \alpha \rho+12 \alpha \rho b n^{2}-4 \alpha \beta-(\alpha-\beta)^{2}}\right)}{2}
\end{aligned}
$$

$\lambda_{3}$

$$
=\frac{\left(\beta-\alpha-j \sqrt{4 a \alpha \rho+12 \alpha \rho b n^{2}-4 \alpha \beta-(\alpha-\beta)^{2}}\right)}{2} .
$$

The stability of the equilibrium can be investigated using Theorem 1: the fractional-order system is asymptotically stable if all the eigenvalues $\lambda_{\Psi}$ of the Jacobian matrix $J_{\Psi}$ satisfy the condition $\left|\arg \left(\lambda_{i}\right)\right|>(\pi / 2) q$ [30]. It determines the stable and unstable regions as shown in Figure 5.

The black region in Figure 5 is the stable region that $\left|\arg \left(\lambda_{i}\right)\right|>(\pi / 2) q$, while light region is the unstable region that $\left|\arg \left(\lambda_{i}\right)\right|>(\pi / 2) q$. When $q=1$, it is the stability of integer-order case.

Here, we consider the simple case $q_{1}=q_{2}=q_{3}=q_{4}=$ $q$, where the fractional-order system has a commensurate order. According to Theorem 2, suppose that the unstable eigenvalues of scroll saddle points are $\lambda_{3,4}=r_{3,4} \pm j \omega_{3,4}$ [30]. The necessary condition to exhibit the chaotic attractor of (9) is the eigenvalues $\lambda_{3,4}$ remaining in the unstable region. The condition for the commensurate derivatives order is $q>$ $(\pi / 2) \operatorname{atan}\left(\left|\omega_{3,4}\right| / r_{3,4}\right)$.

4.2. Phase Portraits. Setting the parameters $a=4, b=0.01$, $\alpha=36, \beta=20, \gamma=3, \rho=3$, and $q=0.85$, the initial value

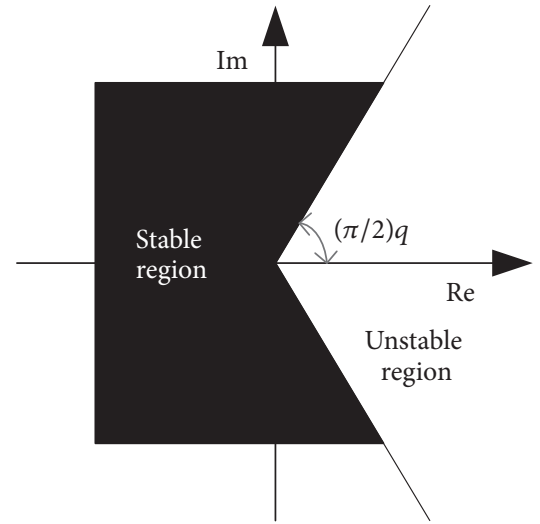

FIGURE 5: Stable and unstable regions in fractional-order 4D hyperchaotic memristive circuit.

of (9) is $(1,0,1,0)$. We get the chaotic attractor as shown in Figure 6. In this case, the Lyapunov exponents of the system are $L_{1}=0.8528, L_{2}=0.3133$, and $L_{3}=0, L_{4}=-59.55$ and the Lyapunov dimension is $d_{L}=3.02$. The fractional-order $4 \mathrm{D}$ hyperchaotic memristive circuit is in a hyperchaotic state as shown in Figure 6. Obviously, in this case, the largest Lyapunov exponent is much bigger than that of integer-order system.

4.3. Lyapunov Exponents Spectra and Bifurcation Diagram. According to the $L E$ spectra calculation algorithm [48], we let $a=4, b=0.01, \alpha=36, \beta=20, \gamma=3$, and $q=0.85$; the initial value of $(9)$ is $(1,0,1,0)$. The Lyapunov exponents spectra and bifurcation diagram of the fractional-order $4 \mathrm{D}$ hyperchaotic memristive circuit by changing $\rho$ simultaneously are shown in Figure 7, where the last Lyapunov exponent is not displayed because it is always a big negative number.

All of the dynamical behaviors of the system with different parameter $\rho$ are summarized in Table 1. It shows that system (9) has abundant dynamical behaviors.

To display its dynamics further, $y-w$ phase portraits of different states with different parameter $\rho$ are presented in Figure 8. With the increase of the parameter $\rho$, the system transforms from hyperchaotic attractor to limit cycle. Obviously, there is a periodic window in the chaotic region when $\rho=(2.0394-2.0407)$.

For above circuit parameters and $\rho=3$, the Lyapunov exponents spectra and bifurcation diagram of the fractionalorder 4D hyperchaotic memristive circuit by changing $q$ simultaneously are shown in Figure 9, where the last Lyapunov exponent is not displayed because it is always a big negative number. The Lyapunov exponents spectra and the bifurcation diagram match very well. Both of them show that the system has complex behaviors. Obviously, once the system is in hyperchaotic state, with the increase of the parameter $q$, the largest Lyapunov exponent becomes smaller and smaller until in the $q=1$. So the fractional-order hyperchaotic system has a higher complexity compared to its corresponding integer-order system. 
TABLE 1: Dynamical behaviors of the system with different $\rho$.

\begin{tabular}{|c|c|c|}
\hline$\rho$ & Sign of Lyapunov exponents & Dynamical behaviors \\
\hline $0 \sim 2.03$ & $+00-$ & Chaos \\
\hline 2.04 & $00--$ & Period 2 cycle \\
\hline $2.05 \sim 2.34$ & $+00-$ & chaos \\
\hline 2.35 & $++0-$ & Hyperchaos \\
\hline $2.36 \sim 2.45$ & $+00-$ & Chaos \\
\hline 2.46 & $++0-$ & Hyperchaos \\
\hline $2.47 \sim 2.51$ & $+00-$ & Chao \\
\hline 2.52 & $++0-$ & Hyperchaos \\
\hline $2.53 \sim 2.55$ & $+00-$ & Chaos \\
\hline 2.56 & $++0-$ & Hyperchaos \\
\hline $2.57 \sim 2.65$ & $+00-$ & Chaos \\
\hline $2.66 \sim 3.08$ & $++0-$ & Hyperchaos \\
\hline 3.09 & $+00-$ & chaos \\
\hline $3.1 \sim 3.2$ & $0---$ & Limit cycle \\
\hline
\end{tabular}

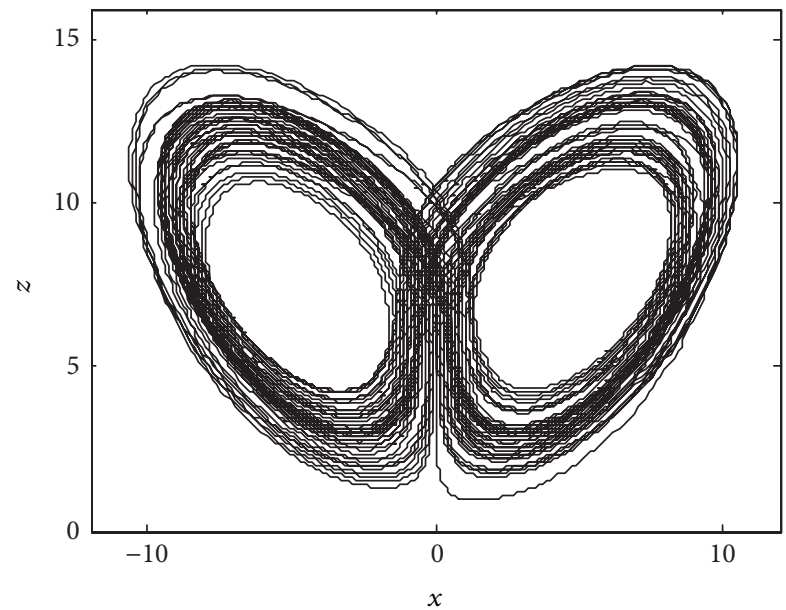

(a) $x-z$

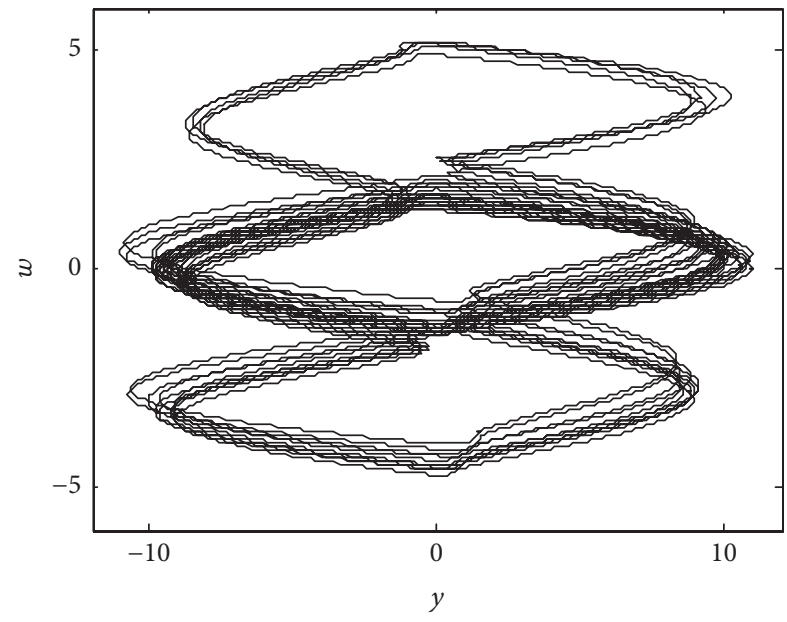

(b) $y-w$

FIGURE 6: Chaotic attractor of the circuit with the 4D hyperchaotic memristive circuit.

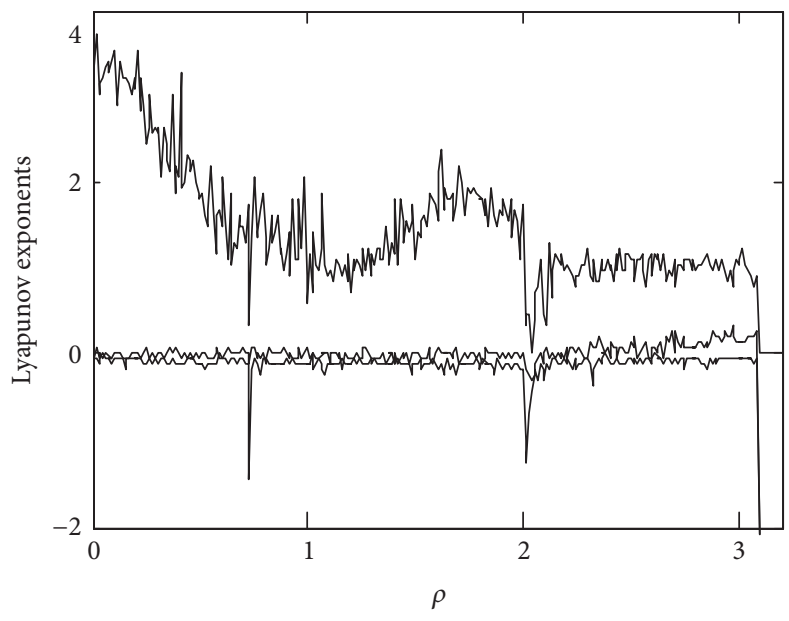

(a) Lyapunov exponents spectrum

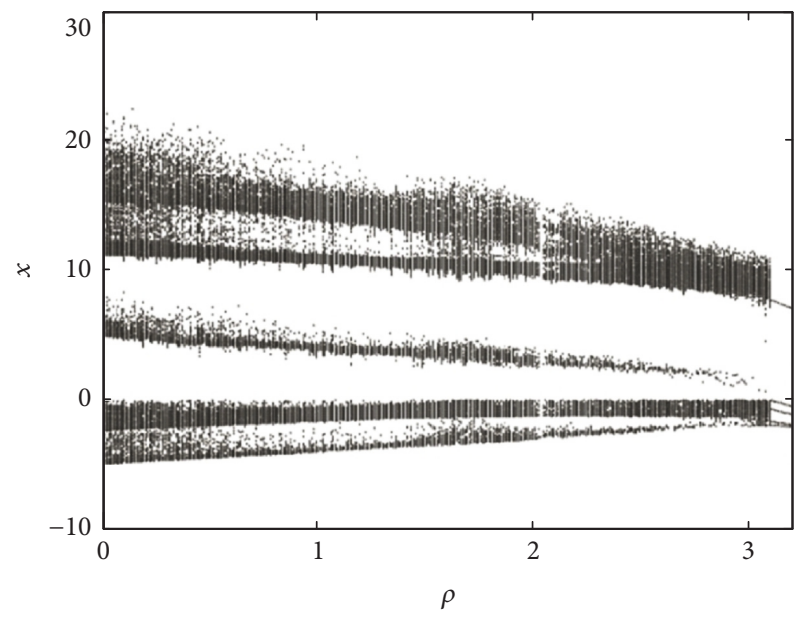

(b) Bifurcation diagram

FIGURE 7: Lyapunov exponents spectrum and bifurcation diagram with variation of the circuit parameter $\rho$. 


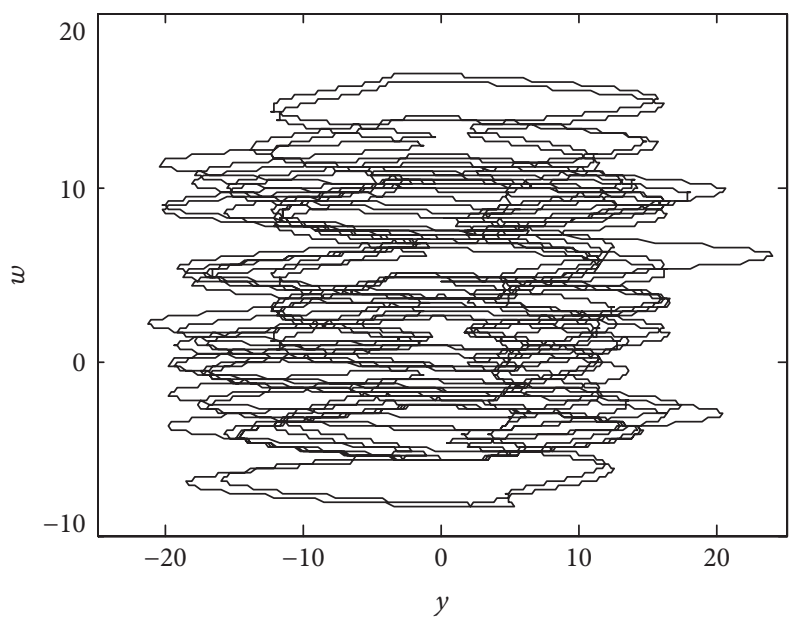

(a) Chaotic attractor $(\rho=0.1)$

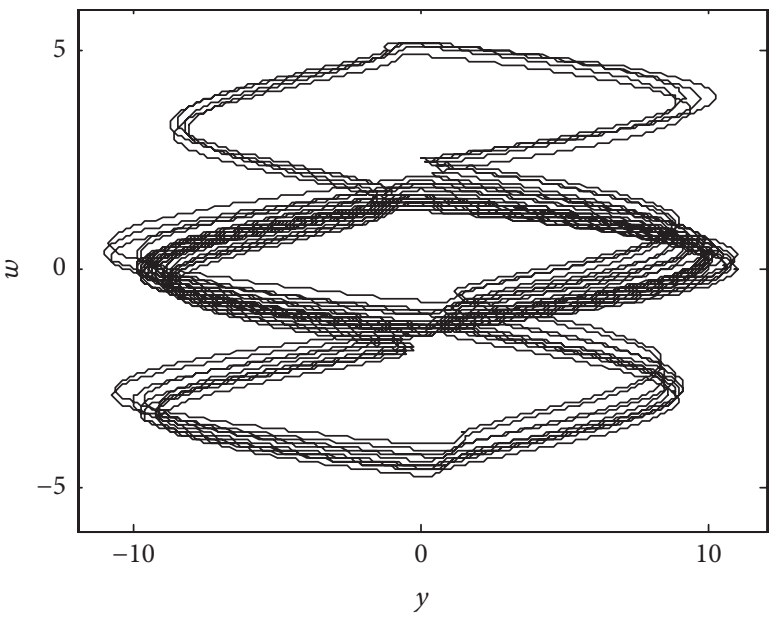

(c) Hyperchaotic attractor $(\rho=3)$

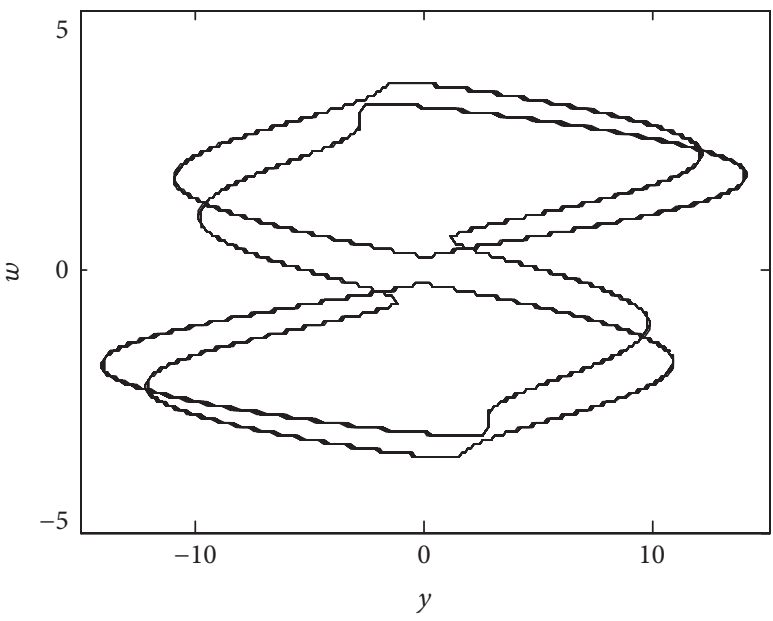

(b) Period 2 cycle $(\rho=2.04)$

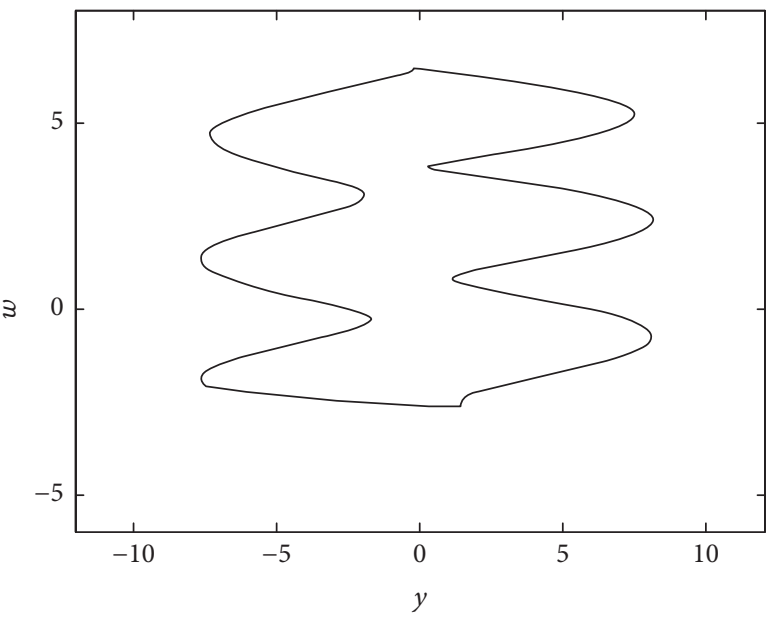

(d) Limit cycle $(\rho=3.15)$

FIGURE 8: Phase portrait with different circuit parameter $\rho$.

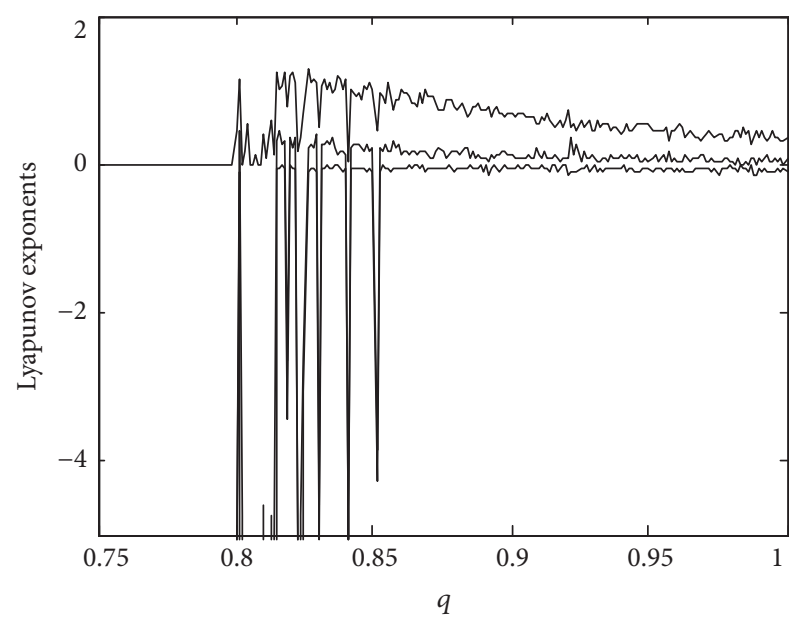

(a) Lyapunov exponents spectrum

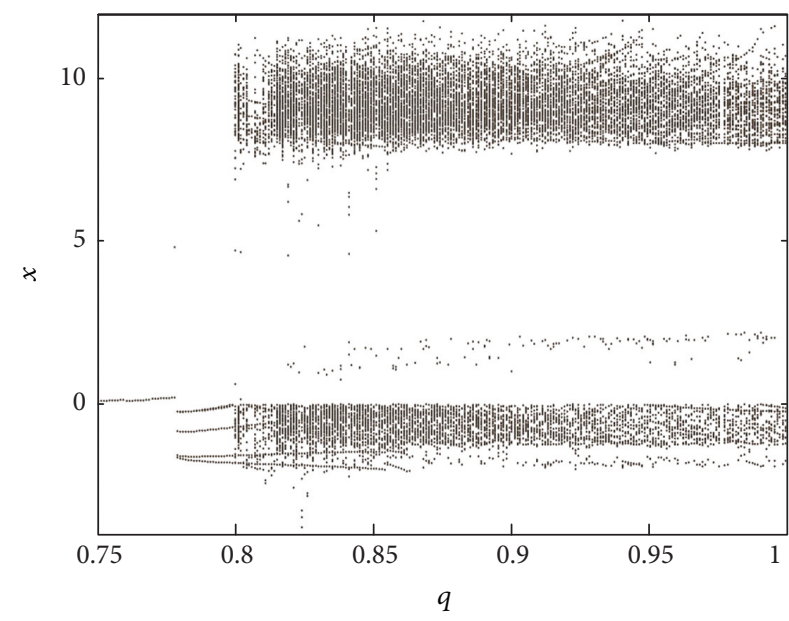

(b) Bifurcation diagram

FIGURE 9: Lyapunov exponents spectrum and bifurcation diagram with variation of the circuit parameter $q$. 
TABLE 2: Dynamical behaviors of the system with different $q$.

\begin{tabular}{lcc}
\hline$q$ & Signs of Lyapunov exponents & Dynamical behaviors \\
\hline $0.75 \sim 0.778$ & $0---$ & Sink \\
$0.779 \sim 0.799$ & $0---$ & Type 1 periodic orbit \\
$0.8 \sim 0.801$ & $+0--$ & Four-scroll chaos \\
0.802 & $0---$ & Type 1 periodic orbit \\
$0.803 \sim 0.804$ & $+0--$ & Four-scroll chaos \\
$0.805 \sim 0.806$ & $0---$ & Type 2 periodic orbit \\
0.807 & $+0--$ & Four-scroll chaos \\
$0.808 \sim 0.809$ & $0---$ & Type 2 periodic orbit \\
$0.81 \sim 0.814$ & $+0--$ & Four-scroll chaos \\
$0.815 \sim 0.818$ & $++0-$ & Three scroll hyperchaos \\
0.819 & $+0--$ & Four-scroll chaos \\
$0.82 \sim 0.822$ & $++0-$ & Three-scroll hyperchaos \\
$0.823 \sim 0.825$ & $+0--$ & Four-scroll chaos \\
$0.826 \sim 0.829$ & $++0-$ & Three-scroll hyperchaos \\
0.83 & $+0--$ & Four-scroll chaos \\
$0.831 \sim 0.84$ & $++0-$ & Three-scroll hyperchaos \\
0.841 & $+0--$ & Four-scroll chaos \\
$0.842 \sim 0.85$ & $++0-$ & Three-scroll hyperchaos \\
0.851 & $+00-$ & Two-scroll chaos \\
$0.852 \sim 1$ & $++0-$ & Two-scroll hyperchaos \\
\hline
\end{tabular}

All of the dynamical behaviors of the system with different parameter $q$ are summarized in Table 2 . It shows that system (9) has abundant and complex dynamical behaviors.

To further display its dynamics, $y-w$ phase portraits of different states with different parameter $q$ are presented in Figure 10. The route to limit cycle from sink can be observed. With the increase of the parameter $q$, the system transforms from chaotic attractor to hyperchaotic attractor. Obviously, there are several periodic windows in the chaotic region.

4.4. Spectral Entropy (SE) and $C_{0}$ Complexity. Complexity measure is an important reference to measure dynamics of a chaotic system. If a chaotic system is used in information security, it can reflect the security of the system to some extent. At present, there are several algorithms for measuring the complexity of chaotic sequences, including intensive statistical [49], multiscale entropy (MSE) [50], spectral entropy (SE) algorithm [51], and $C_{0}$ complexity arithmetic [52]. Among them, $C_{0}$ and SE complexity algorithms have less parameters, faster calculation speed, and higher accuracy. So we employ the SE algorithm to measure the complexity of the fractional-order $4 \mathrm{D}$ hyperchaotic system.

Let $a=4, b=0.01, \alpha=36, \beta=20, \gamma=3$, and $q=0.85$; the initial value of $(9)$ is $(1,0,1,0)$. According to the arithmetic spectral entropy (SE) and $C_{0}$ complexity arithmetic, the SE and $C_{0}$ complexity of the fractional-order $4 \mathrm{D}$ hyperchaotic memristive circuit by changing $q$ and $\rho$ simultaneously are shown in Figure 11.

Figures 7, 9, and 11 show that the Lyapunov exponents spectrum, bifurcation diagram, and complexity are consistent. Figures 11(a) and 11(b) show that the change trends of SE complexity and $C_{0}$ complexity are consistent, but the values are different. It is mainly because of the differences defined by the $\mathrm{SE}$ and $C_{0}$ definitions, which mean that complexity can also reflect the dynamic characteristics of a chaotic system.

\section{Conclusions}

In this paper, the dynamical characteristics of integer-order 4D hyperchaotic memristive circuit are analyzed firstly. Based on Adomian decomposition method (ADM), the numerical solution of a fractional-order 4D hyperchaotic memristive system is investigated and all parameters values of the system are determined. The equilibrium points are obtained, and the distribution of stable and unstable regions of the system is determined. Dynamical characteristics are studied by phase portraits, Lyapunov exponents spectrum, bifurcation diagram, SE complexity, and $C_{0}$ complexity. We found that the dynamical characteristics of fractional-order $4 \mathrm{D}$ hyperchaotic memristive system are more complex than that of the integer-order system, and the SE and $C_{0}$ complexity could reflect the dynamical characteristics quite well. According to the variation of the system dynamics, it is found that the fractional-order hyperchaotic system has better application prospect than its corresponding integer-order chaotic system in the field of chaotic secure communication. Next, we will try to study its implementation in hardware.

\section{Conflicts of Interest}

The authors declare that there are no conflicts of interest regarding the publication of this paper. 


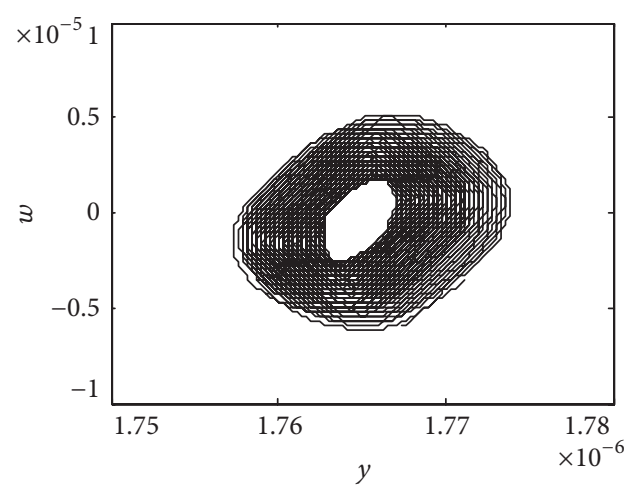

(a) $\operatorname{Sink}(q=0.76)$

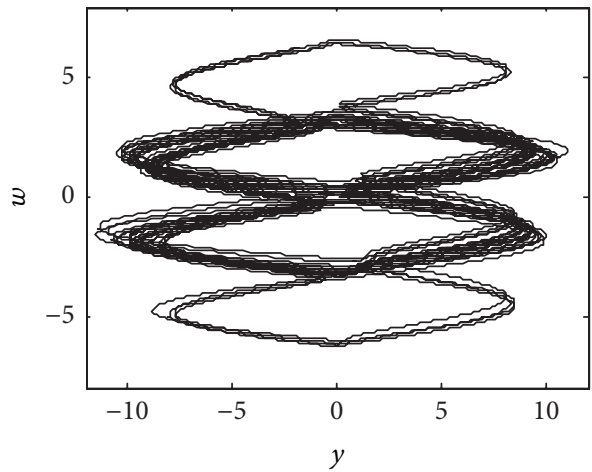

(c) Four-scroll chaotic attractor $(q=0.801)$

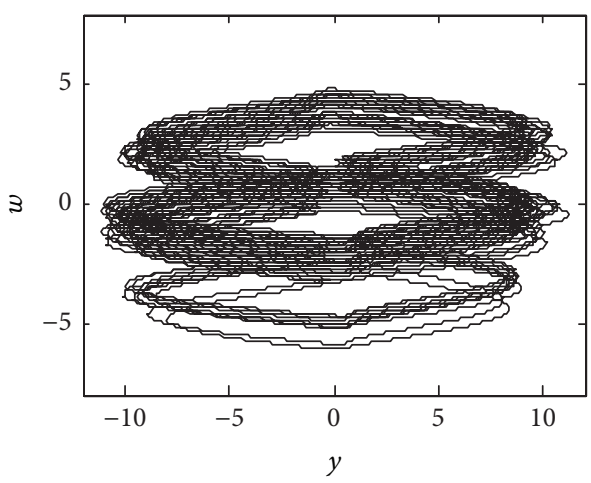

(e) Three-scroll hyperchaotic attractor $(q=0.816)$

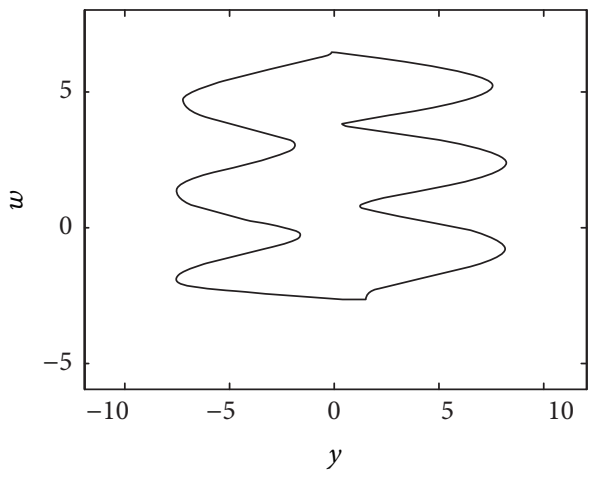

(b) Type 1 limit cycle $(q=0.78)$

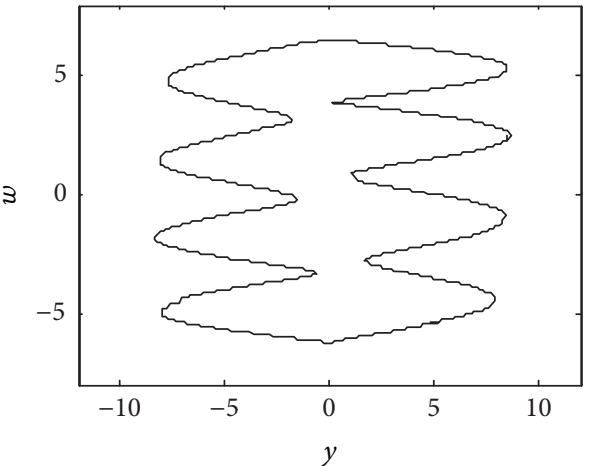

(d) Type 2 limit cycle $(q=0.806)$

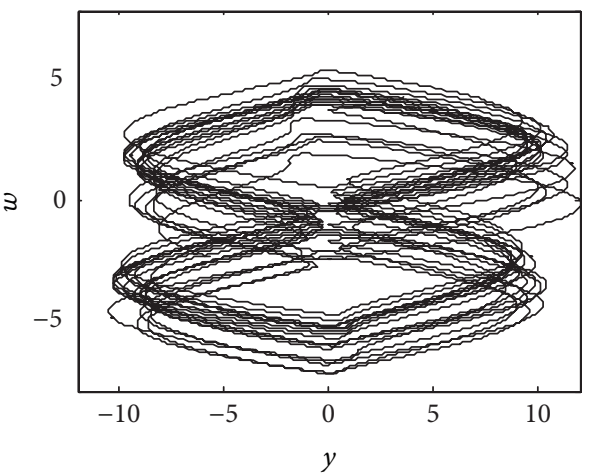

(f) Two-scroll chaotic attractor $(q=0.851)$

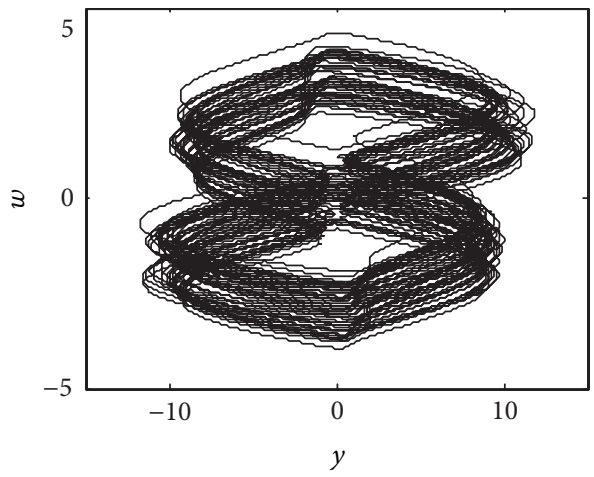

(g) Two-scroll hyperchaotic attractor $(q=1)$

FIGURE 10: Phase portrait with different circuit parameter $q$. 


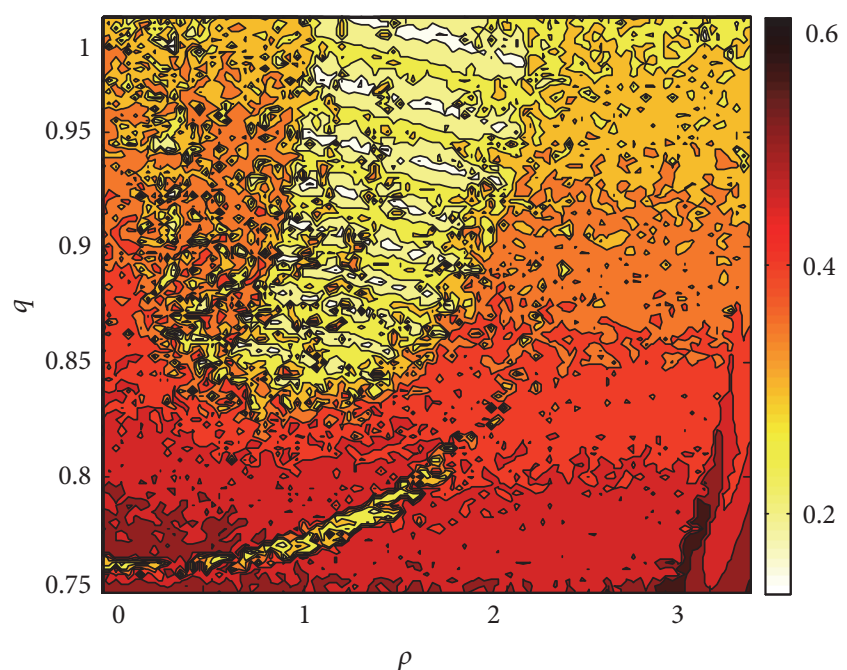

(a) SE complexity

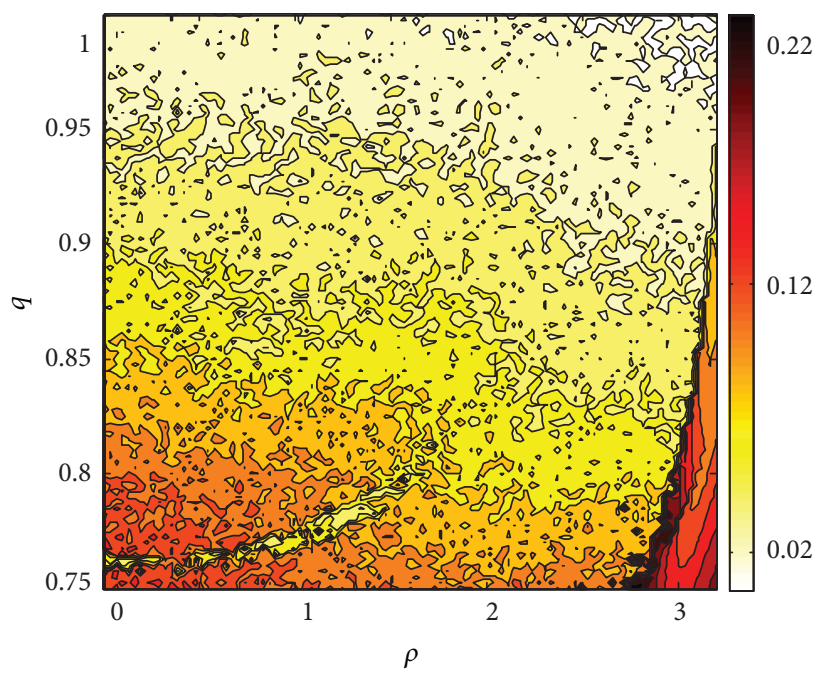

(b) $C_{0}$ complexity

FIGURE 11: SE and $C_{0}$ complexity of the fractional-order $4 \mathrm{D}$ hyperchaotic memristive circuit by changing $q$ and $\rho$.

\section{Acknowledgments}

This work supported by the National Natural Science Foundation of China (Grant nos. 61161006 and 61573383) and Provincial Natural Science Foundation of Liaoning (Grant no. 20170540060).

\section{References}

[1] L. O. Chua, "Memristor-the missing circuit element," IEEE Transactions on Circuit Theory, vol. 18, no. 5, pp. 507-519, 1971.

[2] L. O. Chua and S. M. Kang, "Memristive Devices and Systems," Proceedings of the IEEE, vol. 64, no. 2, pp. 209-223, 1976.

[3] J. M. Tour and H. Tao, "Electronics: the fourth element," Nature, vol. 453, no. 7191, pp. 42-43, 2008.

[4] D. B. Strukov, G. S. Snider, D. R. Stewart, and R. S. Williams, "The missing memristor found," Nature, vol. 453, pp. 80-83, 2008.

[5] F. Corinto, A. Ascoli, and M. Gilli, "Nonlinear dynamics of memristor oscillators," IEEE Transactions on Circuits and Systems. I. Regular Papers, vol. 58, no. 6, pp. 1323-1336, 2011.

[6] L. Teng, H. C. Herbert, and X. Wang, "Chaotic behavior in fractional-order memristor-based simplest chaotic circuit using fourth degree polynomial," Nonlinear Dynamics, vol. 77, no. 1-2, pp. 231-241, 2014.

[7] H. Kim, M. P. Sah, C. Yang, S. Cho, and L. O. Chua, "Memristor emulator for memristor circuit applications," IEEE Transactions on Circuits and Systems. I. Regular Papers, vol. 59, no. 10, pp. 2422-2431, 2012.

[8] M. Dhamala, Y.-C. Lai, and E. J. Kostelich, "Analyses of transient chaotic time series," Physical Review E - Statistical, Nonlinear, and Soft Matter Physics, vol. 64, no. 5, Article ID 056207, pp. 056207/1-056207/9, 2001.

[9] M. Itoh and L. O. Chua, "Memristor oscillators," International Journal of Bifurcation and Chaos in Applied Sciences and Engineering, vol. 18, no. 11, pp. 3183-3206, 2008.
[10] B. Muthuswamy and L. O. Chua, "Simplest chaotic circuit," International Journal of Bifurcation and Chaos, vol. 20, no. 5, pp. 1567-1580, 2010.

[11] B. Muthuswamy, "Implementing memristor based chaotic circuits," International Journal of Bifurcation and Chaos, vol. 20, no. 5, pp. 1335-1350, 2010.

[12] B. Muthuswamy and P. Kokate, "Memristor-based chaotic circuits," IETE Technical Review, vol. 26, no. 6, pp. 415-426, 2009.

[13] B. C. Bao, Z. Liu, and J. P. Xu, "Steady periodic memristor oscillator with transient chaotic behaviours," Electronics Letters, vol. 46, no. 3, pp. 228-230, 2010.

[14] B.-C. Bao, J.-P. Xu, and Z. Liu, "Initial state dependent dynamical behaviors in a memristor based chaotic circuit," Chinese Physics Letters, vol. 27, no. 7, Article ID 070504, 2010.

[15] R. Kilic, Practical Guide for Studying Chua's Circuits, World Scientific, 2010.

[16] W. Xu and X. Yue, "Global analyses of crisis and stochastic bifurcation in the hardening Helmholtz-Duffing oscillator," Science China Technological Sciences, vol. 53, no. 3, pp. 664-673, 2010.

[17] B. Bao, T. Jiang, G. Wang, P. Jin, H. Bao, and M. Chen, "Twomemristor-based Chua's hyperchaotic circuit with plane equilibrium and its extreme multistability," Nonlinear Dynamics, pp. $1-15,2017$.

[18] B. C. Bao, N. Wang, Q. Xu et al., "A simple third-order memristive band pass filter chaotic circuit," IEEE Transactions on Circuits and Systems II Express Briefs, p. 99, 2016.

[19] B. C. Bao, H. Bao, N. Wang et al., "Hidden extreme multistability in memristive hyperchaotic system," Chaos Solitons and Fractals, vol. 94, pp. 102-111, 2017.

[20] H. Wu, B. Bao, Z. Liu, Q. Xu, and P. Jiang, "Chaotic and periodic bursting phenomena in a memristive Wien-bridge oscillator," Nonlinear Dynamics, vol. 83, no. 1-2, pp. 893-903, 2016.

[21] B. Zhang and F. Q. Deng, "Double-compound synchronization of six memristor-based Lorenz systems," Nonlinear Dynamics, vol. 77, no. 4, pp. 1519-1530, 2014. 
[22] B. Bao, G. Shi, J. Xu, Z. Liu, and S. Pan, "Dynamics analysis of chaotic circuit with two memristors," Science China Technological Sciences, vol. 54, no. 8, pp. 2180-2187, 2011.

[23] J. Mou, K. Sun, J. Ruan, and S. He, "A nonlinear circuit with two memcapacitors," Nonlinear Dynamics, vol. 86, no. 3, pp. 1-10, 2016.

[24] F. Yang Y, J. Leng L, and D. Li Q, “The 4-dimensional hyperchaotic memristive circuit based on Chua's circuit," Acta Physica Sinica, vol. 63, no. 8, article 80502, 2014.

[25] A. Wu, "Hyperchaos synchronization of memristor oscillator system via combination scheme," Advances in Difference Equations, 2014:86, 11 pages, 2014.

[26] J. Ruan Y, K. Sun H, and J. Mou, "Memristor-based Lorenz hyper-chaotic system and its circuit implementation," Acta Physica Sinica, vol. 65, no. 19, article 190502, 2016.

[27] J. Sabatier, O. P. Agrawal, and J. A. T. Machado, "Advances in fractional calculus, Theoretical developments and applications in physics and engineering," Biochemical Journal, vol. 361, no. 1, pp. 97-103, 2007.

[28] R. Hilfer, Applications of Fractional Calculus in Physics, World Scientific, Singapore, 2000.

[29] V. Rico-Ramirez, J. Martinez-Lizardo, G. A. Iglesias-Silva, S. Hernandez-Castro, and U. M. Diwekar, "A fractional calculus application to biological reactive systems," Computer Aided Chemical Engineering, vol. 30, no. 4, pp. 1302-1306, 2012.

[30] I. Petras, "Fractional-order memristor-based Chua's circuit," IEEE Transactions on Circuits and Systems II: Express Briefs, vol. 57, no. 12, pp. 975-979, 2011.

[31] I. Petras, "Chaos in fractional-order population model," International Journal of Bifurcation and Chaos, vol. 22, no. 4, Article ID 1250072, 2012.

[32] T. Hartley, C. F. Lorenzo, and H. K. Qammer, "Chaos in a fractional order Chua's system," IEEE Transactions on Circuits and Systems I Fundamental Theory and Applications, vol. 42, no. 8, pp. 485-490, 1995.

[33] D. Cafagna and G. Grassi, "An effective method for detecting chaos in fractional-order systems," International Journal of Bifurcation and Chaos, vol. 20, no. 3, pp. 669-678, 2010.

[34] K. Diethelm, "An algorithm for the numerical solution of differential equations of fractional order," Electronic Transactions on Numerical Analysis, vol. 5, no. 3, pp. 1-6, 1998.

[35] G. Adomian, "A new approach to nonlinear partial differential equations," Journal of Mathematical Analysis and Applications, vol. 102, no. 2, pp. 420-434, 1984.

[36] M. S. Tavazoei and M. Haeri, "Unreliability of frequencydomain approximation in recognising chaos in fractional-order systems," IET Signal Processing, vol. 1, no. 4, pp. 171-181, 2007.

[37] M. S. Tavazoei and M. Haeri, "Limitations of frequency domain approximation for detecting chaos in fractional order systems," Nonlinear Analysis, vol. 69, no. 4, pp. 1299-1320, 2008.

[38] D. Cafagna and G. Grassi, "Bifurcation and chaos in the fractional-order Chen system via a time-domain approach," International Journal of Bifurcation and Chaos in Applied Sciences and Engineering, vol. 18, no. 7, pp. 1845-1863, 2008.

[39] D. Cafagna and G. Grassi, "Hyperchaos in the fractional-order Roussler system with lowest-order," International Journal of Bifurcation and Chaos, vol. 19, no. 1, pp. 339-347, 2011.

[40] S. B. He, K. H. Sun, and H. H. Wang, "Solution of the fractionalorder chaotic system based on Adomain decomposition algorithm and its complexity analysis," Acta Physica Sinica, vol. 63, no. 3, Article ID 030502, 2014.
[41] Q. Li, H. Zeng, and J. Li, "Hyperchaos in a 4D memristive circuit with infinitely many stable equilibria," Nonlinear Dynamics, vol. 79, no. 4, pp. 2295-2308, 2015.

[42] E. F. D. Goufo, "Application of the Caputo-Fabrizio fractional derivative without singular kernel to Korteweg-de VriesBurgers equation," Mathematical Modelling and Analysis, vol. 21, no. 2, pp. 188-198, 2016.

[43] R. Almeida, "A Caputo fractional derivative of a function with respect to another function," Communications in Nonlinear Science and Numerical Simulation, vol. 44, pp. 460-481, 2017.

[44] S. Momani and K. Al-Khaled, "Numerical solutions for systems of fractional differential equations by the decomposition method," Applied Mathematics and Computation, vol. 162, no. 3, pp. 1351-1365, 2005.

[45] V. Daftardar-Gejji and H. Jafari, "Adomian decomposition: a tool for solving a system of fractional differential equations," Journal of Mathematical Analysis and Applications, vol. 301, no. 2, pp. 508-518, 2005.

[46] N. T. Shawagfeh, "Analytical approximate solutions for nonlinear fractional differential equations," Applied Mathematics and Computation, vol. 131, no. 2-3, pp. 517-529, 2002.

[47] R. Gorenflo and F. Mainardi, Fractal and Fractional Calculus in Continuum Mechanics, Springer-Verlag, 1997.

[48] H. Wang, K. Sun, and S. He, "Characteristic analysis and DSP realization of fractional-order simplified Lorenz system based on Adomian decomposition method," International Journal of Bifurcation and Chaos in Applied Sciences and Engineering, vol. 25, no. 6, Article ID 1550085, 1550085, 13 pages, 2015.

[49] H. A. Larrondo, C. M. González, M. T. Martín, A. Plastino, and O. A. Rosso, "Intensive statistical complexity measure of pseudorandom number generators," Physica A: Statistical Mechanics and its Applications, vol. 356, no. 1, pp. 133-138, 2005.

[50] M. Borowiec, A. Rysak, D. N. Betts, C. R. Bowen, H. A. Kim, and G. Litak, "Complex response of a bistable laminated plate: multiscale entropy analysis," European Physical Journal Plus, vol. 129, no. 10, article no. 211, pp. 1-7, 2014.

[51] K. H. Sun, S. B. He, Y. He, and L. Z. Yin, "Complexity analysis of chaotic pseudo-random sequences based on spectral entropy algorithm," Acta Physica Sinica, vol. 62, no. 1, pp. 709-712, 2013.

[52] K.-H. Sun, S.-B. He, C.-X. Zhu, and Y. He, "Analysis of chaotic complexity characteristics based on $\mathrm{C} 0$ algorithm," Acta Electronica Sinica, vol. 41, no. 9, pp. 1765-1771, 2013. 


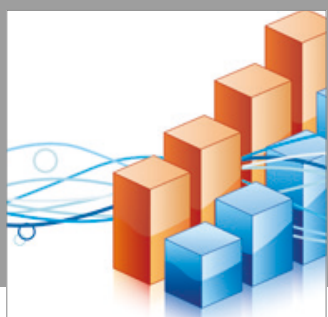

Advances in

Operations Research

vatersals

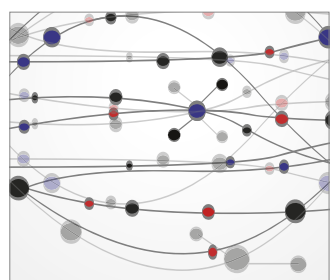

\section{The Scientific} World Journal
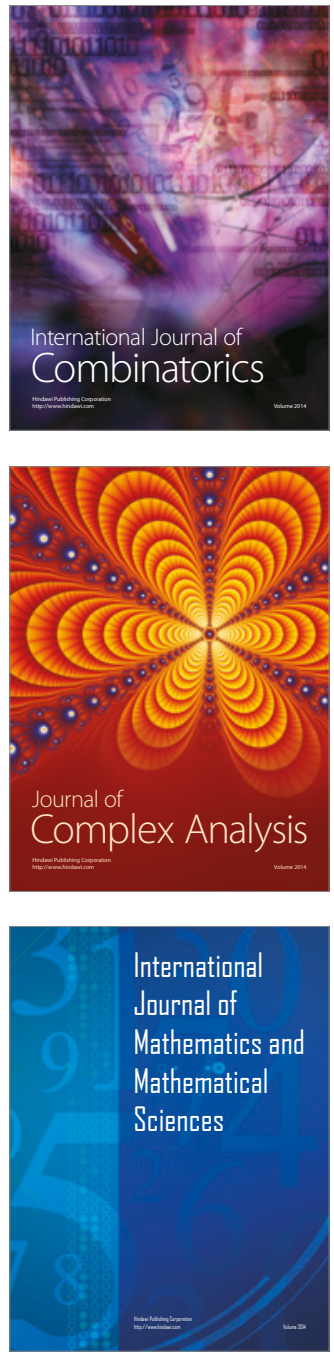
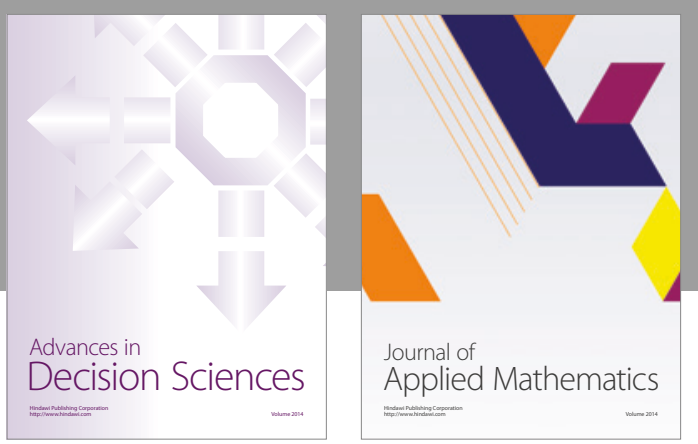

Algebra

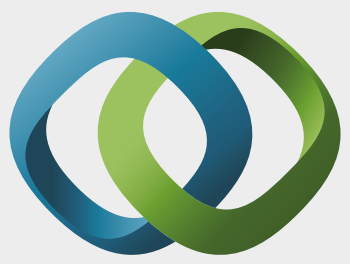

\section{Hindawi}

Submit your manuscripts at

https://www.hindawi.com
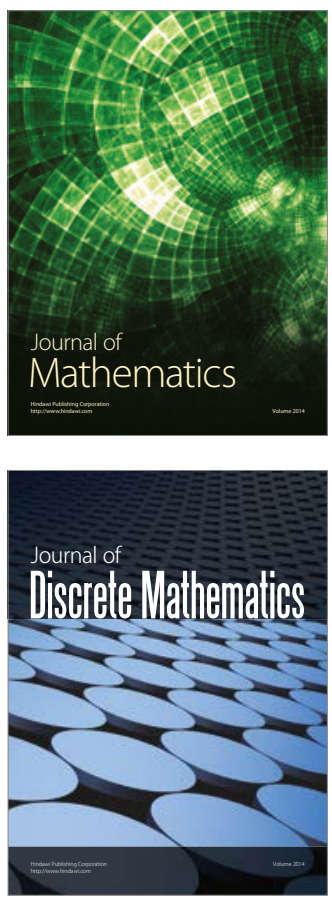

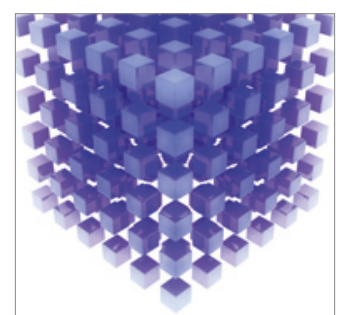

Mathematical Problems in Engineering
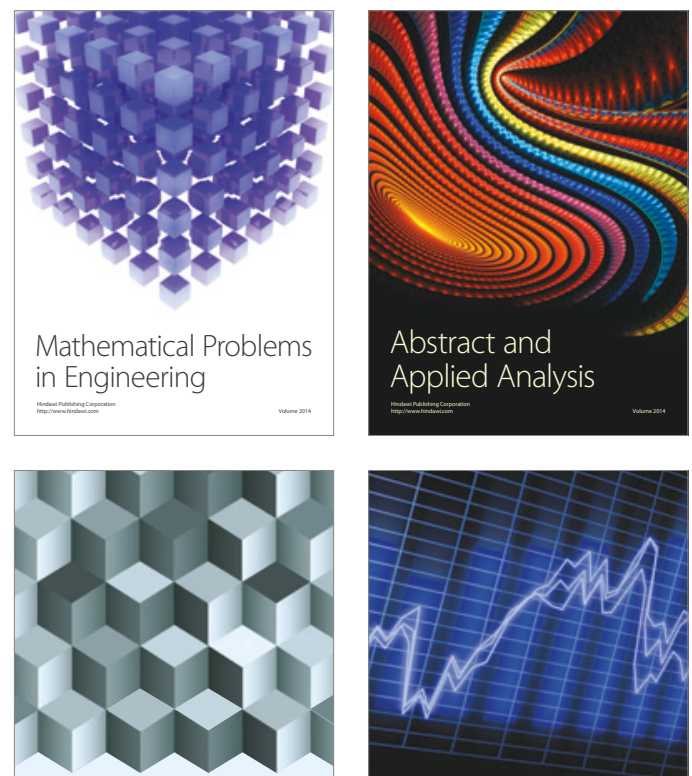

Journal of

Function Spaces

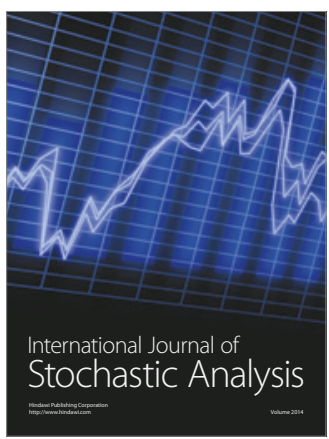

Probability and Statistics
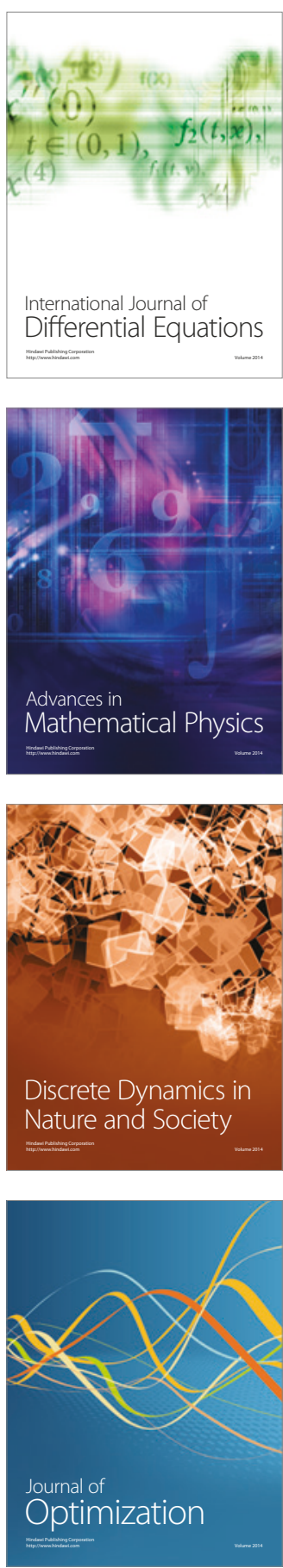